\title{
Vortices in layered superconductors with columnar pins: A density-functional study
}

\author{
Chandan Dasgupta* \\ Centre for Condensed Matter Theory, Department of Physics, Indian Institute of Science, Bangalore 560012, India \\ Oriol T. Valls ${ }^{\dagger}$ \\ School of Physics and Astronomy and Minnesota Supercomputer Institute, University of Minnesota, \\ Minneapolis, Minnesota 55455-0149
}

\begin{abstract}
We use numerical minimization of a model free energy functional to study the effects of columnar pinning centers on the structure and thermodynamics of a system of pancake vortices in the mixed phase of highly anisotropic layered superconductors. The magnetic field and the columnar pins are assumed to be perpendicular to the layers. Our methods allow us to study in detail the density distribution of vortices in real space. We present results for the dependence of the average number of vortices trapped at a pinning center on temperature and pinning strength, and for the effective interaction between nearby pinned vortices arising from short-range correlations in the vortex liquid. For a commensurate, periodic array of pinning centers, we find a line of first order vortex lattice melting transitions in the temperature $T$ vs pin concentration $c$ plane, which terminates at an experimentally accessible critical point as $c$ is increased. Beyond this point, the transition is replaced by a crossover. Our results should also apply, with little change, to thin-film superconductors with strong point pinning.
\end{abstract}

\section{INTRODUCTION}

In the mixed phase of type-II superconductors, magnetic flux penetrates the sample as quantized vortex lines which form a special physical system known as "vortex matter." The fascinating equilibrium and dynamical properties of vortex matter in the mixed phase of high-temperature superconductors (HTSC's) have prompted considerable experimental and theoretical attention ${ }^{1}$ for more than a decade. Because of enhanced thermal fluctuations, the Abrikosov lattice in very pure samples of these highly anisotropic, layered materials is observed to undergo a first order melting transition ${ }^{1}$ into a resistive vortex liquid as the temperature is increased.

The properties of the mixed phase of HTSC's are also known $^{1}$ to be strongly affected by the presence of pinning centers, either intrinsic to the sample or artificially generated. Understanding the effects of pinning in these systems is very important for practical applications because the presence of pinning strongly influences the value of the critical current in the mixed phase. Columnar pinning arising from damage tracks produced by heavy-ion bombardment has received much attention in this context because such extended defects parallel to the direction of the average magnetic flux are highly effective $e^{2,3}$ in increasing the critical current by localizing vortex lines along their length. Columnar defects produce "strong pinning" in the sense that the pinning potential of a defect is sufficiently strong to pin a vortex line at low temperatures. Heavy-ion irradiation generally produces a random array of parallel columnar defects. The effects of such an array of extended defects on the properties of the mixed phase of HTSC's have been extensively studied experimentally, ${ }^{2-5}$ theoretically, ${ }^{6-8}$ and numerically. ${ }^{9,10}$ The thermodynamics of a collection of vortex lines in the presence of a parallel array of random columnar pins has been analyzed ${ }^{6,8}$ by mapping the problem to the quantum mechan- ics of a system of two-dimensional interacting bosons in an external random potential. The main prediction of such theories is the existence of a low-temperature "Bose glass" phase, ${ }^{6-8}$ separated by a continuous phase transition from a high-temperature, entangled liquid of vortex lines. The theoretically predicted scaling behavior near the Bose glass transition has been observed. ${ }^{5}$ A random array of columnar pins also affects the equilibrium properties of the hightemperature vortex liquid, leading to the occurrence of anomalies $^{11}$ in the reversible magnetization curve near $B$ $=B_{\phi}$, where $B_{\phi}=\rho_{p} \Phi_{0}$ ( $\rho_{p}$ is the areal density of columnar pins and $\Phi_{0}=h c / 2 e$ is the flux quantum) is the so-called "matching field," and $B$ is the magnetic induction that determines the areal density $\rho_{0}$ of vortex lines $\left(\rho_{0}=B / \Phi_{0}\right)$.

It is also possible, through the use of a variety of nanofabrication techniques, ${ }^{12-17}$ to create periodic arrays of strong (in the abovementioned sense) pinning centers in thinfilm superconductors. The interplay between the lattice constant of the pin array (determined by $B_{\phi}$ ) and the intervortex separation (determined by $B$ ) can lead to a variety of interesting commensurability effects in such systems. Some of these effects have been observed in recent experiments. Imaging experiments ${ }^{13,17}$ have shown the formation of ordered structures of the vortex system at low temperatures for commensurate values of $B / B_{\phi}$. Magnetization measurements ${ }^{12}$ in the irreversible (vortex solid) regime have demonstrated the occurrence of anomalies at harmonics of $B_{\phi}$. The effectiveness of pinning at integral values of $B / B_{\phi}$ has been found ${ }^{14}$ to produce regularly spaced sharp minima in the resistivity versus field curve. A pinning-induced reconfiguration of the vortex lattice has been observed in an experiment $^{16}$ on a thin-film superconductor with a rectangular array of pinning centers. Some of these effects have been studied theoretically, using analytic ${ }^{18}$ and numerical ${ }^{19}$ methods. Bulk HTSC samples with periodic arrays of columnar 
pins have not been fabricated yet, but the technology for doing this appears to be within reach. ${ }^{20}$

A periodic array of strong pinning centers should have significant effects on the melting transition of the vortex lattice. We consider here the situation where $B>B_{\phi}$, that is, when the pin array is relatively dilute. If, in addition, the value of $B$ is such that the melting temperature of the vortex lattice in the pure system is substantially lower than the superconducting transition temperature in zero field, then each pinning center would pin a vortex at temperatures comparable to the melting temperature of the pure vortex lattice. However, the interstitial vortices, which would be present whenever the number of pinning centers is smaller than the number of vortices (assuming that each defect can pin at most one vortex), may undergo a sharp melting transition. This would certainly be the case in the limit where the spacing of the pin array is sufficiently large. Since the vortices pinned at the defects produce a periodic potential for the interstitial ones, the melting transition of the latter is an example of a solid to liquid transition in the presence of an external periodic potential. Evidence for such melting of interstitial vortices has been found in experiments ${ }^{12,13}$ on thinfilm superconductors with periodic pinning. However, the thermodynamic behavior at the melting transition has not been characterized in these experiments. The effects of a periodic potential on the melting of two-dimensional solids have been investigated earlier using analytic ${ }^{21}$ and numerical ${ }^{22,23}$ methods. We are not aware of any theoretical study of the effects of a periodic array of columnar pins on the vortex lattice melting transition in three dimensions.

In this paper, we report the results of a study of the equilibrium properties of the mixed phase of highly anisotropic, layered superconductors in the presence of columnar pins. We consider a geometry in which both the magnetic field and the direction of the columnar defects are perpendicular to the superconducting layers. Our study is based on a model free energy functional ${ }^{24-26}$ for a system of "pancake" vortices lying on the superconducting layers. We consider the limiting case of a vanishingly small Josephson coupling between the layers, so that the pancake vortices on different layers interact via only their electromagnetic coupling. As we discuss in some detail early in the next section, previous theoretical and experimental studie ${ }^{25-30}$ have shown that this limit is appropriate for describing extremely anisotropic ${ }^{31} \mathrm{Bi}$ - and $\mathrm{Tl}-$ based HTSC's. These are the systems that we will consider here. The Ramakrishnan-Yussouff (RY) free-energy functional ${ }^{24}$ used in the present work is the same as that used in earlier studies ${ }^{25,26}$ of vortex lattice melting in pure systems. The same free energy functional was also used, in combination with the replica method for treating quenched disorder, in a study ${ }^{32}$ of the effects of random point pinning on the melting line in the $B-T$ plane. In these earlier studies, the density distribution in the crystalline state was expressed in terms of a few "order parameters" and the free energy was minimized with respect to these parameters. In the present work, we use a different method which is more powerful and more appropriate for describing in detail pinninginduced inhomogeneities of the local density. This method, developed in our studies ${ }^{33}$ of the hard-sphere system, in- volves direct numerical minimization of a discretized version of the free energy functional. Since both the magnetic field and the direction of the columnar pins are assumed to be perpendicular to the layers, the time-averaged local density of pancake vortices must be the same on all the layers. This simplification makes the problem effectively twodimensional and allows a high-precision numerical investigation of the effects of columnar pins on the structure and thermodynamics of the vortex system. Furthermore, our results should apply, with little change, to thin film superconductors with strong pinning.

The model considered in our work is defined in Sec. II, where the method of calculation is also described. We then consider (Sec. III A) the effects of an isolated columnar pin on the structure of the vortex liquid in the vicinity of the pin. This is done mainly for testing the systematics of our numerical method and also for determining appropriate values of the pinning potential to be used in subsequent calculations. We choose throughout the computations numerical parameter values appropriate for $\mathrm{BSCCO}$. We determine the suitable choice of the discretization scale in order that our numerical method provides an accurate account of the density inhomogeneities produced by the trapping of a vortex at a pinning center. We also determine the range of values of the pinning potential strength for which nearly one vortex is trapped at a pinning center in the temperature range of interest. The strength of the pinning potential is kept fixed in this range in our subsequent work: pinning of multiple vortices at a pinning center is not considered because this is rarely observed in experiments. Next, in Sec. III B, we consider the effects of two neighboring pinning centers on the liquid-state properties. An "effective interaction" between vortices trapped at the two pinning centers is obtained by calculating the free energy as a function of the separation between the pinning centers. This effective interaction is found to oscillate with distance. This study and the one-pin calculation mentioned above complement, in a sense, the analytic work of Ref. 18 where the RY free-energy functional was used to analyze the structure and magnetization of a twodimensional vortex liquid in the presence of strong pinning. However, we consider here a three-dimensional system with columnar pins, instead of a two-dimensional system with point pinning as in Ref. 18. Also, the numerical direct minimization method used in the present work is more accurate than the analytic variational method in the earlier study.

We next study (Sec. III C) the freezing of the vortex liquid in the pure system. This is done primarily for checking the method against the results of earlier calculations. ${ }^{25,26} \mathrm{We}$ find results in excellent agreement with those of earlier studies. Our method also provides a very detailed and accurate account of the distribution of the density near a lattice point. We then proceed, in Sec. III D, to consider the melting transition of interstitial vortices in a commensurate, triangular array of columnar pins. As discussed above, this transition provides a physical realization of three-dimensional melting in the presence of a commensurate periodic potential. Defining the concentration $c$ of pinning centers as $c \equiv B_{\phi} / B$, we consider values of $c$ given by $1 / l^{2}$ where $l$ is an integer. For small concentrations of pinning centers $(l \geqslant 6)$, we find a 
first-order melting transition from a crystalline solid to an inhomogeneous liquid. As the pin concentration is increased, the transition temperature increases and the latent heat and the jump in the crystalline order parameter at the transition decrease. We find that this line of first-order transitions terminates at a critical point beyond which the thermodynamic transition is replaced by a sharp crossover. This critical point is a rare, experimentally realizable example of continuous melting in three dimensions. We show that a simple Landau theory provides a semiquantitative understanding of most of our results. Some of our most salient results on the melting transition in the presence of a periodic pin array were summarized in a recent paper. ${ }^{34}$ Here, we present many details which could not be included in that short paper. Section IV contains a summary of the main results and some concluding remarks.

\section{MODEL AND METHODS}

As explained in the Introduction, we perform in this paper a numerical study using density-functional theory, which involves, as its foundation, a model free energy functional appropriate to a system of pancake vortices in a layered superconductor. Density-functional methods have long been used $^{24,35}$ with great success in the study of solidification phenomena in ordinary fluid systems. Although the theory is basically mean-field based, it works very accurately in the description of first-order melting. We have ourselves performed extensive numerical studies of a density vs disorder strength phase diagram of a hard sphere system in the presence of quenched disorder ${ }^{33}$ using a methodology quite similar to that employed in the present work. Density functional methods have also been successfully used ${ }^{25,26}$ to study the melting of the vortex lattice in layered superconductors without pinning.

The starting point of our calculation is an expression for the free energy of the system, written as a functional of the time averaged local density. In our case the relevant density is $\rho(i, \mathbf{r})$, the time averaged areal density of pancake vortices at point $\mathbf{r}$ on the $i$ th layer. In the homogeneous vortex liquid state in the absence of pinning, this density is uniform and it is given in terms of the magnetic induction $B$ by $\rho_{0}=B / \Phi_{0}$, where $\Phi_{0}$ is the superconducting flux quantum. It is customary and convenient to introduce a length $a_{0}$ through the relation $\pi a_{0}^{2} \rho_{0}=1$. We will use $a_{0}$ as our standard unit of length in terms of which other lengths will be given and we will usually also normalize densities in terms of $\rho_{0}$.

We write the free energy functional in the form

$$
F[\rho]=F_{\mathrm{RY}}[\rho]+F_{p}[\rho]
$$

The first term in the right-hand side of Eq. (2.1) is the free energy of the vortex system in the absence of pinning, while the second includes the pinning effects. Since the potential produced by a collection of straight columnar pins perpendicular to the layers is the same on every layer, the timeaveraged density of vortices at any point $\mathbf{r}$ must be the same on all layers, i.e., $\rho(i, \mathbf{r})=\rho(\mathbf{r})$ for all $i$. Then, the free en- ergy per layer corresponding to the first term in the right side of Eq. (2.1) may be written ${ }^{24}$ in an effectively twodimensional form

$$
\begin{aligned}
\beta F_{\mathrm{RY}}[\rho]= & \int d \mathbf{r}\left\{\rho(\mathbf{r}) \ln \left[\rho(\mathbf{r}) / \rho_{0}\right]-\delta \rho(\mathbf{r})\right\} \\
& -(1 / 2) \int d \mathbf{r} \int d \mathbf{r}^{\prime} \widetilde{C}\left(\left|\mathbf{r}-\mathbf{r}^{\prime}\right|\right) \delta \rho(\mathbf{r}) \delta \rho\left(\mathbf{r}^{\prime}\right),
\end{aligned}
$$

where $\beta$ is the inverse temperature. We have defined $\delta \rho(\mathbf{r})$ $\equiv \rho(\mathbf{r})-\rho_{0}$ as the deviation of $\rho(\mathbf{r})$ from $\rho_{0}$, the density of the uniform liquid and taken our zero of the free energy at its uniform liquid value. The function $\widetilde{C}(r)$ is a static correlation function that contains all the required information about the interactions in the system. It is given by $\Sigma_{n} C(n, r)$ where $n$ is the label denoting layer separation, $r$ is the in-plane distance, and $C(n, r)$ is the direct pair correlation function ${ }^{35}$ of a layered liquid of pancake vortices with areal density $\rho_{0}$.

Strongly anisotropic layered superconductors can be described in terms of the Lawrence-Doniach ${ }^{36}$ Hamiltonian from which the energy of a system of pancake vortices can be derived. ${ }^{37}$ In general, these vortices interact via a combination of electromagnetic and Josephson couplings. To calculate the contribution of the Josephson coupling to the energy, one needs to specify, in addition to the positions of the vortices on the superconducting layers, the precise way in which these vortices are to be connected to form lines. The resulting effective "Hamiltonian" of the vortex system cannot be written as a sum of pairwise interactions. However in the limit of infinite anisotropy, which corresponds to vanishingly small Josephson coupling, the pancake vortices interact via a pairwise potential which is nonzero for vortices lying on different layers because they continue to be coupled through their electromagnetic interaction. This limit is an appropriate starting point for describing BSCCO, for which the anisotropy factor $\gamma \equiv \lambda_{c} / \lambda_{a b}\left(\lambda_{c}\right.$ and $\lambda_{a b}$ are the penetration depths for currents flowing perpendicular and parallel to the copper-oxide planes, respectively) has been estimated ${ }^{31}$ to be higher than 500. The regime where Josephson coupling is relevant is given by ${ }^{28} \lambda_{a b}>\gamma d$ where $d$ is the layer spacing. Since $\lambda_{a b}(T=0) \sim 1500 \AA$ and $d \simeq 15 \AA$ for BSCCO, $\lambda_{a b}(T=0)$ is substantially smaller than $\gamma d$, which indicates $^{28}$ that the electromagnetic coupling should dominate the behavior of the vortex system over much of the $H-T$ plane.

Analytic $^{25,26}$ and numerical ${ }^{27}$ studies based on the approximation of infinite anisotropy have yielded results in good agreement with those obtained from experiments on BSCCO. Also, several experimental studies ${ }^{29,30}$ have shown that in BSCCO, the electromagnetic interaction between vortices dominates over the interaction generated by the Josephson coupling if the temperature is lower than about $0.8 T_{c}(H=0)$. As the low-temperature behavior will be our primary concern, the assumption of infinite anisotropy is quite reasonable for BSCCO. However, this approximation should break down in less anisotropic systems such as 
YBCO where the Josephson coupling can not be neglected over much of the $H-T$ plane. ${ }^{28}$

In the limit of infinite anisotropy, it is relatively straightforward ${ }^{26}$ to calculate $C(n, r)$ and $\widetilde{C}(r)$ using the hypernetted chain (HNC) approximation. ${ }^{35}$ Since the interaction term in Eq. (2.2) is of a convolution form, it is numerically most efficient to deal with it in wave vector space. This and the use of fast Fourier transform (FFT) methods reduces the computation of the interaction term in the free energy to a single sum. One begins with the expression for the Fourier transform of $v(n, r)$, the two-body vortex-vortex interaction, which, in the limit of vanishingly small Josephson coupling between the layers, is given ${ }^{25,26}$ by

$$
\beta v(\mathbf{k})=\frac{2 \pi \Gamma \lambda^{2}\left[k_{\perp}^{2}+\left(4 / d^{2}\right) \sin ^{2}\left(k_{z} d / 2\right)\right]}{k_{\perp}^{2}\left[1+\lambda^{2} k_{\perp}^{2}+4\left(\lambda^{2} / d^{2}\right) \sin ^{2}\left(k_{z} d / 2\right)\right]},
$$

where $k_{z}$ and $k_{\perp}$ are, respectively, the components of $\mathbf{k}$ perpendicular and parallel to the layer plane, $d$ is the layer spacing, and $\lambda(T)$ the penetration depth in the layer plane. The dimensionless quantity $\Gamma$ which determines the strength of the interactions is given ${ }^{38}$ by

$$
\Gamma=\beta d \Phi_{0}^{2} / 8 \pi^{2} \lambda^{2} .
$$

In coordinate space, $v(0, r)$ is repulsive and logarithmic in $r$ while $v(n \neq 0, r)$ is also logarithmic, but attractive and weaker than the intralayer potential by a factor of roughly $(d / \lambda) e^{-n d / \lambda}$. Beginning with an interaction of this form, the HNC procedure of Ref. 26 can be used to numerically compute $C(\mathbf{k})$ for the appropriate values of the relevant parameters. The quantity $\widetilde{C}\left(k_{\perp}\right)$, the two-dimensional Fourier transform of $\widetilde{C}(r)$, is then obtained by setting $k_{z}=0$ in $C(\mathbf{k})$.

The second term in Eq. (2.1) represents the contribution of pinning to the free energy per layer. It is given by

$$
\beta F_{p}[\rho]=\int d \mathbf{r} V_{p}(\mathbf{r}) \delta \rho(\mathbf{r}),
$$

where $V_{p}(\mathbf{r})$ is the dimensionless (normalized by $k_{B} T$ ) pinning potential at point $\mathbf{r}$. This quantity can be written as $V_{p}(\mathbf{r})=\Sigma_{j} v_{p}\left(\left|\mathbf{r}-\mathbf{R}_{j}\right|\right)$, where the sum is over all pinning centers located at the points $\left\{\mathbf{R}_{j}\right\}$ on a plane, and $v_{p}(r)$ is the dimensionless form of the potential at $\mathbf{r}$ due to a pinning center at the origin. We take this potential to be of the truncated parabolic form ${ }^{18}$

$$
v_{p}(r)=-V_{0}\left[1-\left(r / r_{0}\right)^{2}\right] \theta\left(r_{0}-r\right),
$$

where $r_{0}$ is the range of the pinning potential. We will write the dimensionless strength parameter $V_{0}$ as $V_{0}=\alpha \Gamma$ and the quantity $\alpha$ will be chosen, as explained below, so that the pinning is strong enough to localize one vortex at a pinning center at the temperatures of interest, but not so strong that more than one vortex is bound to a pinning center.

In order to carry out numerical work, we have to discretize our system. We introduce for this purpose a computational triangular lattice of size $L$. On the sites of this lattice we define density variables $\rho_{i} \equiv \rho\left(\mathbf{r}_{i}\right) v$, where $\rho\left(\mathbf{r}_{i}\right)$ is the density at mesh point $i$ and $v$ the area of the unit cell in the computational lattice, proportional to the square of its lattice constant $h$. We have $L \equiv N h$, so that the computational lattice has $N^{2}$ sites. Periodic boundary conditions are used in all our calculations.

Our basic procedure is to minimize the free energy of the system given certain values of the relevant parameters and the appropriate initial conditions, that is, some initial set of values for the computational variable $\rho_{i}$. Finding the minima of the free energy is not at all trivial, since one is minimizing a function of a very large number of variables (we have used values of $N$ up to 2048 as discussed below) and these variables $\rho_{i}$ can take values differing by many orders of magnitude (particularly in the solid phase) and must also satisfy the nonnegativity constraint. This precludes the use of many standard minimization methods. The procedure we use here is the same as that originally employed in the hard sphere problem ${ }^{33,39,40}$ with the important difference that the calculations involving the interacting term are performed in wave vector space. This is for two reasons: first, it turns out to be much more efficient, since the time used by Fourier transforming back and forth using efficient FFT routines turns out to be negligible compared with the time saved by not having to perform a double sum over the large computational lattice. Second, the direct correlation function is more conveniently computed in any case in terms of the wave vector variable. The procedure we use incorporates ${ }^{39}$ the nonnegativity constraints and is insensitive to the large range of the variables, but requires ${ }^{33}$ a large number of iterations for convergence. The efficiency of the FFT method, however, still allows us to use the sizes required for the problem.

The minimization procedure finds a local minimum of the free energy. The minimum located depends on the initial values chosen for the set of variables $\rho_{i}$. The appropriate choices in each case are discussed in the next section. Generally speaking, nearly uniform initial conditions lead to liquid minima while ordered initial conditions with the proper symmetry lead to crystalline states.

\section{RESULTS}

\section{A. General and one pin}

In this section we present and discuss our numerical results. In principle, these could be given in terms of a minimal set of dimensionless parameters. However, it is more appropriate, in view of this paper's objectives as discussed in the Introduction, to present the results in terms of physical parameters with dimensions. This is the course we have taken. The values of the material parameters that we use here have been therefore chosen as appropriate to BSCCO. These parameters are the penetration depth $\lambda$ and the interplane distance $d$, which together with the temperature and fundamental constants determine $\Gamma$. We set $d=15.26 \AA, \lambda(T=0)$ $=1500 \AA$, and assume a two-fluid temperature dependence of $\lambda(T)$ with $T_{c}(H=0)=85 \mathrm{~K}$. For these parameter values, the dimensionless quantity $\Gamma \simeq 2650 /(T$ in $\mathrm{K})$ at low temperatures where the $T$ dependence of $\lambda$ is negligible. We study temperatures and fields in the region where the melting transition of the vortex lattice is expected to occur. The strength of the pinning potential is described by the param- 


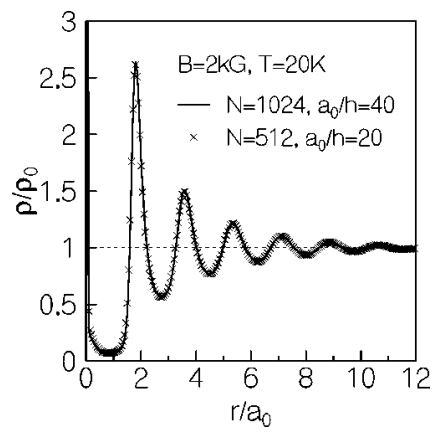

FIG. 1. Numerical accuracy of density profile calculations. The normalized local density variable $\rho / \rho_{0}$ in the presence of a single pinning center is plotted vs $r$, the distance from the pinning center in units of $a_{0}$. Two sets of data are plotted, for the same physical parameter values $(B=2 \mathrm{kG}, T=20 \mathrm{~K})$. One set was computed with $N=512, a_{0} / h=20$ (crosses) and the other set (solid line) with $N=1024, a_{0} / h=40$ (see text). A third set with $N=2048, a_{0} / h$ $=80$ would be completely obscured by the solid curve if plotted.

eter $\alpha \equiv V_{0} / \Gamma$, as introduced above. We fix the range $r_{0}$ to $r_{0}=0.1 a_{0}$, which corresponds to about $55 \AA$ for $B=2 \mathrm{kG}$.

To test our procedures and to find out more about the parameter range to be studied and the system sizes required, we begin by considering the simple case of an isolated pinning center in a vortex system in the liquid state. We place this pinning center in the center of the computational lattice. Since periodic boundary conditions are used, this amounts to considering a periodic array of pinning centers with spacing equal to the size $L$ of the computational cell. As discussed below, the values of $L$ used in these studies are sufficiently large, so that the behavior near a pinning center is not affected by the presence of its periodic images. We then choose the initial configuration of the variables $\rho_{i}$ as one vortex located at the pinning center and uniform density everywhere else, with the average density consistent with $\rho_{0}$. We then perform the minimization of the free energy as described above. The main issues here are the determination of the appropriate values of the pinning strength, and, from a technical standpoint, finding the system sizes, and the values of $h / a_{0}$ required. A smaller value of $h / a_{0}$ implies higher spatial resolution in describing the variation of the local density, but at constant $N$ this amounts to a reduction in the size of the system being studied. One must therefore strike a balance.

We have performed this procedure for fields $B=2$ and 3 $\mathrm{kG}$ and at several temperatures in the range of interest (15 $-22 \mathrm{~K})$ at those fields. We have considered values of $N$ ranging from 128 to 2048 with $a_{0} / h$ from a maximum of 80 down to values of order unity. Representative results of this rather extensive study are shown in Fig. 1. In this figure we plot the density variable $\rho$ (normalized by $\rho_{0}$ ) as a function of the dimensionless distance $r$ from the pin, measured in units of $a_{0}$. The data in the figure are all taken at a temperature $T=20.0 \mathrm{~K}$ and a field $B=2 \mathrm{kG}$, with the parameter $\alpha$ set at 0.06. Results are shown for two cases: $N=512$ with $a_{0} / h=20$ and $N=1024, a_{0} / h=40$. The scaling of $a_{0} / h$ with $N$ ensures that we are comparing systems containing the same number of vortices. The two results are very close to each other. We have also obtained results for $N=2048$,

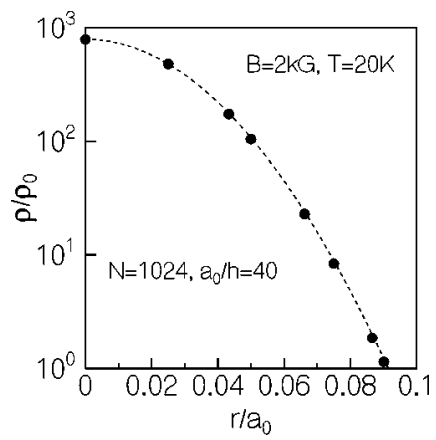

FIG. 2. Short distance (within the range of the pinning potential) results for the normalized density profile (dots). A fit to an exponential form in the pinning potential $v_{p}(r)$ (dotted line, see text) is also shown.

$a_{0} / h=80$, which are completely indistinguishable from those at $N=1024$, so that if we had plotted them they would not be visible: the two plots at $N=1024$ and $N=2048$ would be on top of each other. This and similar data obtained at other temperatures and fields tell us the range of values of $N$ and $a_{0} / h$ needed to obtain high quality data. The results subsequently presented in this and the next subsection are obtained at $N=1024$ and $a_{0} / h=40$

The high peak at small $r$ in Fig. 1 represents the large vortex density at the pinned site. This density then decays away in an oscillatory manner, as shown in the figure, towards its long range limit, which is unity for our normalization. As expected, ${ }^{18}$ the behavior of $\rho(r) / \rho_{0}$ outside the range of the pinning potential is very similar to that of the radial distribution function ${ }^{35}$ of the unpinned vortex liquid at the temperature and magnetic induction being considered. Thus, the medium and long range behavior of the density is reasonable. The behavior of $\rho(r)$ at very short distances, inside the pinning range, is shown in Fig. 2. One can see in this figure how the results are well fit, as expected, by an exponential form $e^{-v_{p}(r)}$, where the pinning potential in units of $k_{B} T$ is given by Eq. (2.6). This confirms that our computational mesh is sufficiently fine even at these very small ranges.

Next, we must find the appropriate values of the pinning strength parameter $\alpha$, as introduced above. We wish to consider here, of course, the case of strong pinning, but nevertheless $\alpha$ should remain small enough so that the total amount of flux pinned at each site remains on the average below one superconducting flux quantum. To choose the appropriate value, we studied the average number of vortices pinned as a function of $\alpha$. Sample results are shown in Fig. 3 , where the number of vortices pinned at the site (obtained by integrating the density over the pinning range) is displayed as a function of $\alpha$. The data shown in this figure were taken at $B=2 \mathrm{kG}$ and $T=18.0 \mathrm{~K}$; data at other relevant fields and temperatures are very similar. We see that the number of pinned vortices rises very rapidly with $\alpha$ until a very marked kink occurs, at about the value when one vortex is pinned on the average. It is clear that one should use a value of $\alpha$ just below the kink in the curve, and in this study we have used the values $\alpha=0.05$ and $\alpha=0.06$ (the smaller value was used in calculations at lower temperatures). For $T$ 


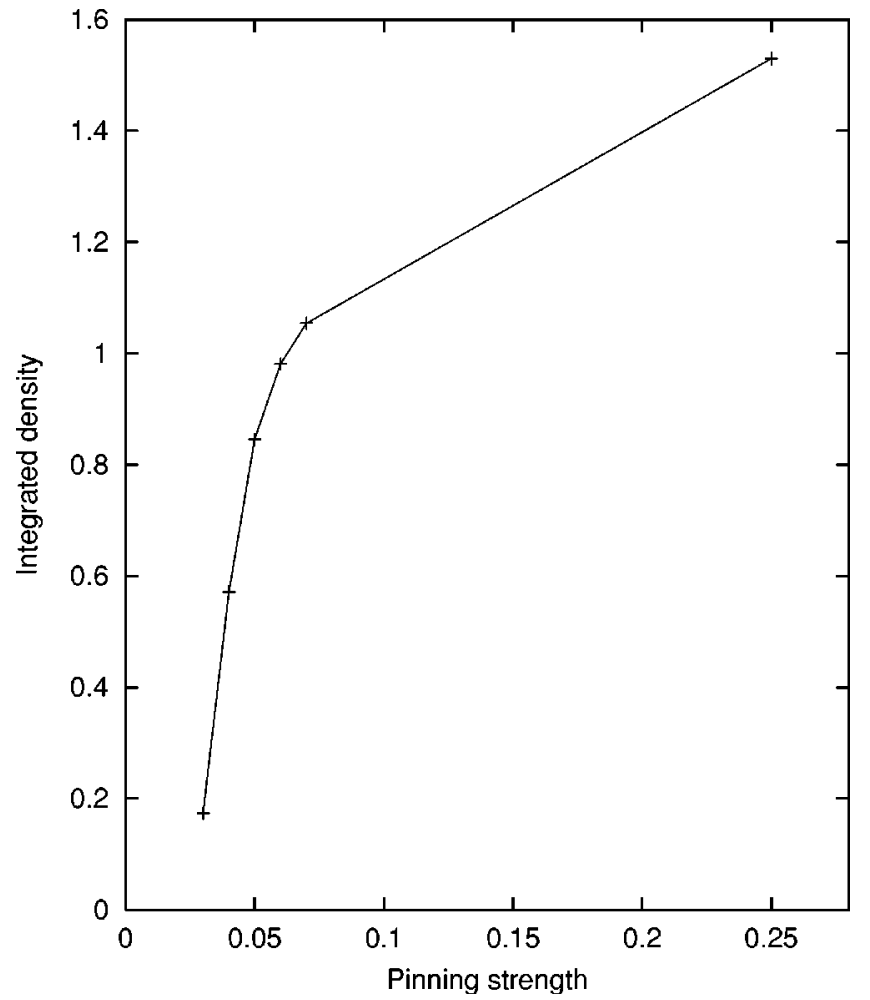

FIG. 3. Integrated density within the range of the pinning potential (that is, the average number of vortices pinned at a center), as a function of pinning strength given by the parameter $\alpha$, defined in the text. For values of the pinning strength just before the kink in the curve, nearly one vortex is pinned at a center. The temperature is $20 \mathrm{~K}$ and the field $2 \mathrm{kG}$.

in the range of interest here $(15-22 \mathrm{~K})$, these values of $\alpha$ correspond to $V_{0} \sim 7-9$. This is consistent with the results of the two-dimensional study of Ref. 18 where it was found that the average number of vortices trapped at a pinning center decreases sharply below one as the dimensionless pinning strength $V_{0}$ becomes lower than about 8 .

\section{B. Two pinning centers}

Having in the previous subsection determined the properties of the density profile when the pins are, in effect, very far apart, we consider now the case where there are two pinning centers separated by a smaller distance $d$. With $\alpha$ set so that each center pins nearly one vortex, we expect to have, when the two centers are not too far apart, interactive effects as a function of $d$, as the density oscillations emanating from each pinning center (see Fig. 1) must be distorted to match each other. We perform this calculation by placing the two pinning centers symmetrically around the center of the computational triangular lattice, on the longer diagonal. For the initial conditions, we place one vortex on each of the two pinning sites, and a uniform density on the remaining computational sites, consistent with the average density being $\rho_{0}$. We have performed this study at fields of 2 and $3 \mathrm{kG}$ and at several temperatures. Results at $B=2 \mathrm{kG}$ and $T=20 \mathrm{~K}$, with $\alpha=0.06$ are shown in Fig. 4. These results were again obtained at $N=1024, a_{0} / h=40$. In the main plot we give

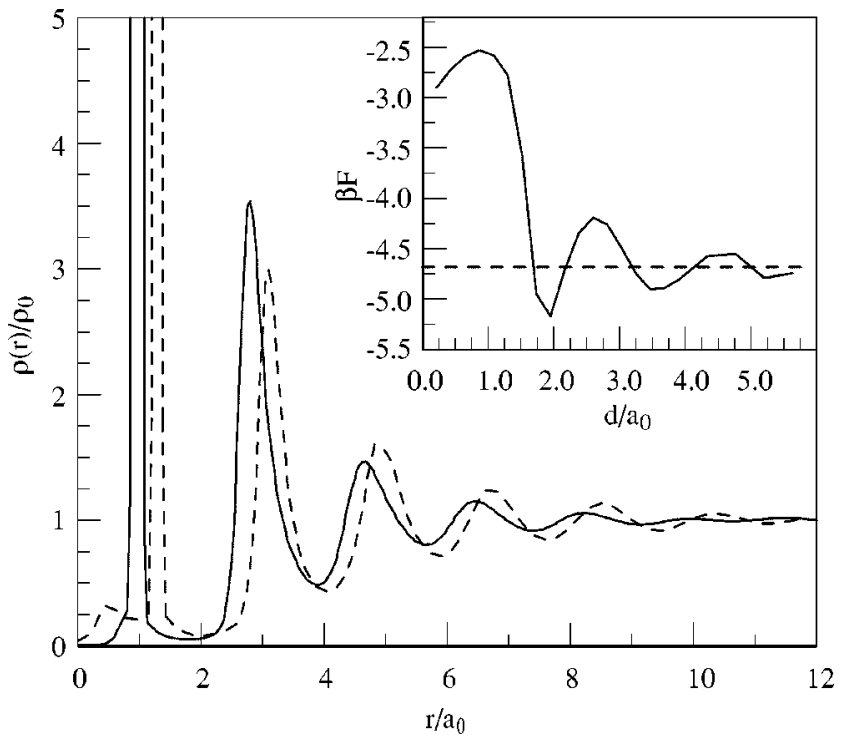

FIG. 4. Density profiles in the presence of two pinning centers. The two centers are placed symmetrically around the origin (see text). In the main plot, the density profiles are shown as a function of the distance $r$ from the origin, that is, from the midpoint between the two pinning sites. The inset shows (solid line) the free energy as a function of the pin separation $d$. The horizontal dashed line marks the infinite distance limit. The curves shown in the main plot are for the cases where the distance $d$ between centers equals $1.95 a_{0}$ (solid curve) and $2.60 a_{0}$ (dashed curve). The first corresponds to a minimum of $\beta F$ (inset), the second to a nearby maximum. The results shown are for $B=2 \mathrm{kG}, T=20 \mathrm{~K}$.

two examples of the density profile as a function of distance. Only one half of the density distribution is plotted, with the origin corresponding to the center of the lattice and the horizontal axis representing distances along the diagonal. The distribution is then symmetric about the origin, and the distance from the center of the first peak to the plot's origin is half the interpin distance. The solid curve corresponds to the case where $d$ (in units of $a_{0}$ ) is 1.95 and the dashed curve to the case where it equals 2.60. One can see at larger values of $r$, away from both pins, an oscillatory decay similar to that in Fig. 1. The behavior in the interpin region near the plot origin is more complicated. At shorter interpin distances (as in the solid curve), the density is very small between the two centers, but when that distance is increased to over twice $a_{0}$ (see the dashed curve), it becomes possible to have a peak between the two pinning centers, and as $d$ is further increased, additional intersite peaks appear as well.

When the two centers are close enough to interact, the free energy will obviously depend on whether the oscillations in the density profiles corresponding to the two centers "lock" or not. This implies ${ }^{18}$ that the free energy of the system should be an oscillatory function of interpin distance. To verify this, we have evaluated the free energy as a function of interpin distance $d$. Results are shown in the corner inset of Fig. 4. The dashed horizontal line is the result for the case where $d$ is very large, that is, twice the value for a single pin [recall that the zero of free energy is taken to be the at the uniform liquid state, see Eq. (2.2)]. This value is $\beta F$ $=-4.680$ for the case plotted. The solid curve shows the 
behavior of $\beta F$ as a function of interpin distance and it clearly displays the oscillatory behavior of this quantity. As found in Ref. 18, the free energy has minima at interpin distances approximately corresponding to the position of the maxima of the single pin density profile shown in Fig. 1. This reflects that it is easier, for those distances, to lock the oscillations corresponding to the two centers. The two pin distances corresponding to the two curves shown in the main plot of Fig. 4 were chosen so that one corresponds to a free energy minimum (solid line) while the other (dashed curve) corresponds to a nearby maximum. The higher value of the free energy in the latter case is due to greater difficulty in matching the two profiles in this case. This difficulty is reflected in the smaller height of the first peak of $\rho(r)$ outside the range of the pinning potential and the appearance of a small peak of $\rho(r)$ near $r / a_{0} \simeq 0.6$. The oscillatory behavior of the free energy as a function of $d$ implies an oscillatory dependence of the magnetization of the vortex liquid on the applied magnetic field when a periodic array of pinning centers is present. In particular, the reversible magnetization in the liquid state is expected to show minima near certain integral values of $B / B_{\phi}$.

The integrated density inside the range of a pinning center remains close to unity when the pin separation $d$ is greater than $2 a_{0}$. As the value of $d$ is decreased below $2 a_{0}$, the integrated density begins to decrease and becomes substantially lower than unity for $d<1.5 a_{0}$. Thus, the simultaneous occupation of two pinning centers by two vortices is likely if the centers are far apart (in units of $a_{0}$ ), but unlikely only when the two pinning centers are separated by distances less than about $1.5 a_{0}$. This is consistent with decoration experiments ${ }^{41}$ which show that nearly all pinning sites are occupied by vortices when the number of pinning sites is smaller than the number of vortices $\left(B>B_{\phi}\right)$.

\section{Melting in the pure system}

As a preliminary step in our study of the effect of an array of pinning centers on vortex lattice melting, we have carried out calculations of the melting transition of the pure system for $B=2$ and $3 \mathrm{kG}$. This is in order to determine the "clean" limit of our subsequent results. In addition, our numerical solutions in the pure limit can be compared with those obtained from variational treatments ${ }^{25,26}$ of the same RY freeenergy functional in which the density distribution in the crystalline phase was expressed in terms of the Fourier components of the density at a few small reciprocal lattice vectors.

The computational cell used in our pure limit calculations is one triangular-lattice unit cell with lattice constant $L$. The spacing $h$ of the computational grid is chosen to have the values $L / N$ with $N=16,32,64$, and 128 . Crystalline minima of the free energy are obtained by running the minimization routine with initial states that have a sharp peak of the density at the center of the computational cell. At sufficiently low temperatures such minima are found for a range of values of $L$. Typical results obtained for $B=2 \mathrm{kG}, L$ $=1.9884 a_{0}$, and two values of $N(N=16$ and $N=128)$ are shown in Fig. 5 where the dimensionless free energy $\beta F$ of

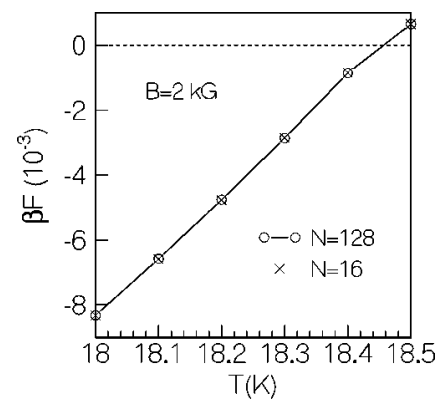

FIG. 5. Computation of the melting temperature for the pure system at a field $B=2 \mathrm{kG}$. The symbols, connected by a solid line, represent the computational results for the dimensionless free energy of the crystal, as explained in the text. The results are shown to be independent of $N$ which determines (see text) the mesh size used in the computation. The temperature at which the solid line crosses the liquid free energy (zero by convention, dashed line) is the melting point.

one unit cell of the vortex crystal is plotted as a function of the temperature $T$. We find that the free energies of the crystal obtained for all the values of $N$ listed above are essentially the same, as exemplified by the data shown for two values of $N$, indicating that the effects of discretization are minimal provided that $h \leqslant L / 16 \simeq 0.125 a_{0}$.

The equilibrium value $L_{0}$ of the lattice parameter is determined by finding the value of $L$ that minimizes the free energy at a given $B$ and $T$. The dependence of $\beta F$ on the value of $L$ for $B=2 \mathrm{kG}, T=18.5 \mathrm{~K}$ is shown in Fig. 6. The value of $L_{0}$ is found to be close to $1.988 a_{0}$, which is slightly higher than the spacing $\sqrt{2 \pi / \sqrt{3}} \quad a_{0}$ of a triangular lattice of density $\rho_{0}$. This reflects the well-known result ${ }^{1}$ that the density of a vortex lattice increases slightly at melting. The transition temperature is determined from the zero crossing of the free energy of the crystalline state, calculated for $L=L_{0}$, as a function of $T$, as illustrated in Fig. 5, which shows the results of computations performed at $L=L_{0}$. For $B=2 \mathrm{kG}$, the melting temperature is then $T_{c}=18.45 \mathrm{~K}$. This value of $T_{c}$ is slightly higher than that obtained variationally. ${ }^{26}$ This is expected: the free energy of the crystal obtained in our unconstrained minimization should be lower than that obtained in calculations where the free energy is minimized with respect to a few parameters only.

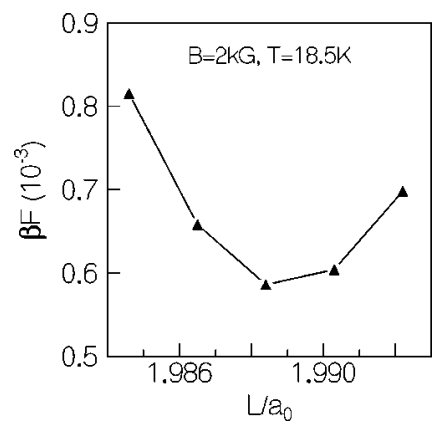

FIG. 6. Determination of the equilibrium lattice parameter $L_{0}$ at melting. The dimensionless free energy of the crystal at fixed $B$ and $T$ is plotted here vs the lattice parameter $L$. The free energy has a minimum at $L=L_{0} \simeq 1.988 a_{0}$. 


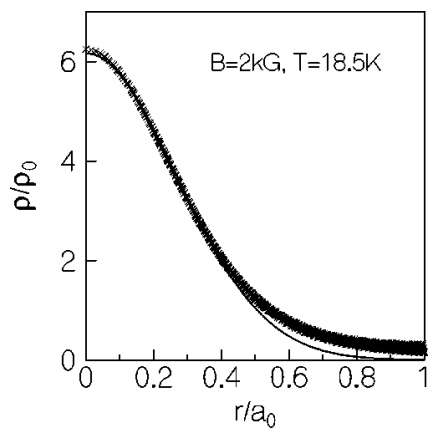

FIG. 7. Radial dependence of the density distribution in the vortex lattice. The quantity plotted is the angular average of the normalized local density $\rho(r) / \rho_{0}$. It is given as a function of the distance from the center of a crystalline unit cell. The symbols (crosses) are the computed results, and the solid line is a Gaussian best fit, valid at small distances from the center of the cell.

The discontinuity in the entropy at the crystallization transition is obtained from the numerically calculated slope of the $\beta F$ versus $T$ curve at the transition temperature. The Fourier transform of the density distribution at the crystalline minimum obtained at the transition temperature yields the value of the jump in the crystalline order parameter $m$, defined as the magnitude of the Fourier component of the density at the shortest reciprocal lattice vector of the triangular lattice. At $B=2 \mathrm{kG}$ the entropy change $\Delta s$ per vortex is $0.29 k_{B}$, and the jump in the order parameter $m$ is $\Delta m$ $=0.52$. Very similar results are obtained for $B=3 \mathrm{kG}: T_{c}$ $=15.10 \mathrm{~K}, \Delta s=0.28 k_{B}, \Delta m=0.52, L_{0}=1.985 a_{0}$. These are in close agreement with the results of earlier studies. ${ }^{1,26}$

Our numerical method provides detailed and accurate information about the spatial distribution of the time averaged density in a unit cell of the vortex lattice. We find that the density function near the center of the unit cell is, to a good approximation, invariant under a rotation about an axis perpendicular to the layers and passing through the center of the unit cell. Figure 7 shows a plot of the local density $\rho(r)$ (angularly averaged and normalized by the average density $\rho_{0}$ ) at the crystalline minimum obtained for $B=2 \mathrm{kG}, T$ $=18.5 \mathrm{~K}, L=1.988 a_{0}$, as a function of the distance $r$ from the center of the unit cell. As shown in the figure, a Gaussian fit to the data for small $r$ provides a good account of the dependence of the density on $r$ except at larger distances. The value of the Lindemann parameter $\mathcal{L}$ at melting may be obtained approximately from the width of the gaussian fit, or more accurately from a numerical evaluation of the rootmean-square displacement

$$
\left\langle r^{2}\right\rangle=\frac{\int r^{2} \rho(\mathbf{r}) d \mathbf{r}}{\int \rho(\mathbf{r}) d \mathbf{r}},
$$

where the integrals are over a lattice unit cell and $\mathbf{r}$ is the radius vector measured from the center of the unit cell. We find that the value of $\mathcal{L}$ at melting is 0.26 for $B=2 \mathrm{kG}$ and 0.25 for $B=3 \mathrm{kG}$. These values, similar to those found in earlier work, ${ }^{1,26}$ are substantially larger than the typical val- ues of $\mathcal{L}$ in simple three-dimensional solids near melting. The large value of $\mathcal{L}$ implies that the peak of the density at the center of the unit cell is not very sharp (see Fig. 7). This helps explain why relatively coarse values of the mesh size $h$ (e.g., $h=L_{0} / 16$ ) are adequate for obtaining an accurate description of the density distribution in the crystalline state.

\section{Periodic array of columnar pins}

Having obtained these clean limit results in the previous subsection, we proceed now with our study of the effects of a commensurate, periodic array of pins on the vortex lattice melting transition. We consider a triangular lattice of pins with spacing equal to $l L_{0}$, where $l$ is an integer and $L_{0}$, as defined above, is the equilibrium value of the spacing of the pure vortex lattice at its melting point for the value of $B$ being considered. Thus the pin concentration is $c \equiv 1 / l^{2}$. The computational cell used is one unit cell of the pin lattice (which contains $l^{2}$ unit cells of the vortex lattice) with periodic boundary conditions and one pin located at the center of one of the vortex lattice unit cells. The value of $h$ was fixed at $L_{0} / 64$ in the calculations for $B=2 \mathrm{kG}$. We also carried out a few calculations for $B=2 \mathrm{kG}$ using $h=L_{0} / 16$. The results obtained for this larger value of $h$ were found to be essentially the same as those obtained for $h=L_{0} / 64$. We therefore used $h=L_{0} / 16$ in our calculations for $B=3 \mathrm{kG}$. We set the pin strength parameter, as mentioned above, to $\alpha=0.06$ in the calculations for $B=2 \mathrm{kG}$. A slightly smaller value, $\alpha=0.05$, was used in the calculations for $B=3 \mathrm{kG}$ because the temperature range of interest is lower in this case and the strength required to pin slightly less than one vortex is somewhat smaller.

The crystalline and liquid minima of the free energy were located from "heating" and "cooling" runs, respectively. In a heating run, the crystalline minimum was first located by minimizing the free energy starting with an initial state in which the density distribution in each of the $l^{2}$ vortex lattice unit cells contained in the computational cell was that in one unit cell of the vortex lattice of the pure system at its melting point for the same value of $B$. The crystalline minimum so obtained was "followed" to higher temperatures by increasing the temperature in small steps and running the minimization program at each temperature with the minimum obtained at the previous step as the initial state. In a cooling run, a liquid minimum was first obtained at a relatively high temperature by minimizing the free energy with an initial state consisting of one vortex located at the pinning center and uniform density everywhere else, so that the average density was $\rho_{0}$. This minimum was then "followed" to lower temperatures as in the heating runs, but decreasing the temperature in small steps instead of increasing it. The liquid state is not homogeneous in the presence of pinning and its free energy is nonzero.

For both values of $B$ studied and relatively small values of $c(l=10,8,7$, and 6$)$, we found a range of temperatures over which both crystalline and liquid minima are locally stable. The crossing of the free energies of these two minima defines a first-order transition between crystalline and liquid states. Results for the transition temperature $T_{c}$ as a function of $c$ 


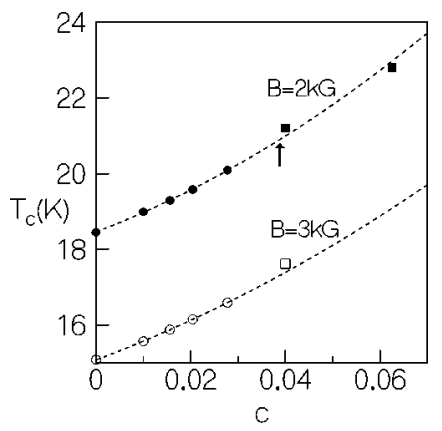

FIG. 8. Phase diagram (transition temperature $T_{c}$ as a function of pin concentration $c$ ) for $B=2 \mathrm{kG}$ (solid symbols) and $B$ $=3 \mathrm{kG}$ (open symbols). The circles denote first order transitions and the squares mark crossovers. The dotted lines are polynomial fits, included to guide the eye. The arrow marks the approximate position of the critical point at $B=2 \mathrm{kG}$. At $B=3 \mathrm{kG}$ the critical point is near $T=17.6 \mathrm{~K}$.

for $B=2$ and $3 \mathrm{kG}$ are shown in Fig. 8. The presence of columnar pins is found to increase $T_{c}$. This should be expected: columnar pins suppress the disordering effects of the lateral wandering of vortex lines, and a commensurate periodic array of such pins clearly promotes crystallization. In other words, an external potential having the same symmetry as the crystal favors the crystalline state. The results for $B$ $=2$ and $3 \mathrm{kG}$ are quite similar, with $T_{c}$ for $B=3 \mathrm{kG}$ reduced by approximately $3.4 \mathrm{~K}$ for all these values of $c$. The discontinuities in the entropy $s$ and the order parameter $m$ decrease as $c$ increases ${ }^{34}$ because pinning-induced order in the liquid increases with $c$.

Our results yield not only bulk quantities but also very detailed information on the density distribution of the vortices. This quantity is experimentally accessible in scanning tunneling microscopy (STM) and scanning Hall probe measurements. In Fig. 9, we show the variation of the local density $\rho$ along a line joining two neighboring pinning centers for the crystalline and liquid minima near the transition temperature for $c=1 / 49$ and $B=2 \mathrm{kG}$. The density profile in the liquid minimum can be viewed as a superposition of liquidlike profiles near individual pins (compare with Fig. 1,

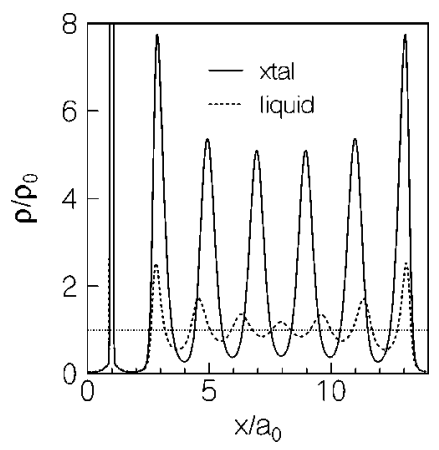

FIG. 9. Density distribution for the coexisting liquid and crystal states at the melting transition for pin concentration $c=1 / 49$ and field $B=2 \mathrm{kG}$. The normalized density profiles are plotted along a line joining two pins, one near $x=0$, and the other just beyond the right edge of the figure.

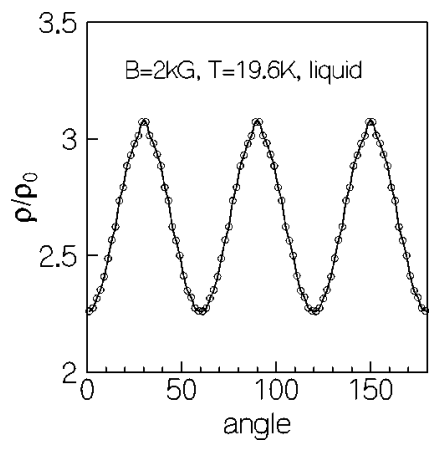

FIG. 10. Sixfold angular modulation of the density in the liquid state for $B=2 \mathrm{kG}, T=19.6 \mathrm{~K}, c=1 / 49$. The normalized density averaged over the first peak (see text) is plotted vs the angle measured from a line joining two pinning sites.

noting the vertical axis scale), with small-amplitude, damped oscillations about $\rho=\rho_{0}$. In contrast, the density in the crystalline state exhibits higher, sharper and asymmetric peaks at the lattice points, with the density rising more sharply on the side closer to the pin, particularly at smaller distances from the pin site. Similar plots for $c=1 / 64$ may be found in Ref. 34 . These plots clearly bring out the obvious differences between the structures of the coexisting crystal and inhomogeneous liquid phases.

The density distributions at the liquid minima exhibit as expected ${ }^{18}$ a sixfold angular modulation. This is illustrated in Fig. 10 where we have shown the average density at the first peak of the normalized local density near a pinning center (i.e., the density averaged over the region $1.75 a_{0} \leqslant r$ $\leqslant 1.9 a_{0}$, where $r$ is the distance from the pinning center) as a function of the angle measured from the line joining the pinning center to one of its nearest neighbors. The data shown are for the liquid minimum obtained for $B=2 \mathrm{kG}$, $T=19.6 \mathrm{~K}$ and $c=1 / 49$. A sixfold angular modulation of the density is clearly seen in the figure. The minima of the density occur on the lines that join the pinning center to its nearest neighbors. This is different from the behavior found in the crystalline minima where density maxima occur on the lines joining neighboring pinning sites (this can be seen at a different value of $c$ from inspection of Fig. 12 below).

The full two-dimensional density distributions at the liquid and crystalline minima obtained near the transition temperature for $B=2 \mathrm{kG}$ and $c=1 / 36$ are shown as gray scale plots in Figs. 11 and 12, respectively. The plot for the liquid minimum exhibits the usual correlation "hole" around the pinning site at the center, and concentric "rings" of alternating high and low densities with sixfold angular modulation. The angular modulation at the first ring is less pronounced (and less obvious in a gray scale plot) than that depicted in Fig. 10 for $c=1 / 49$. The plot for the crystalline minimum illustrates how the detailed structure of the periodically arranged crystalline density peaks changes with the distance from the pinning site at the center. The ability of our numerical method to provide detailed information about the density distribution in highly inhomogeneous states is clearly illustrated in these figures as well as in Figs. 9 and 10.

The degree of order in the liquid state increases with the pin concentration $c$, thereby decreasing the difference be- 


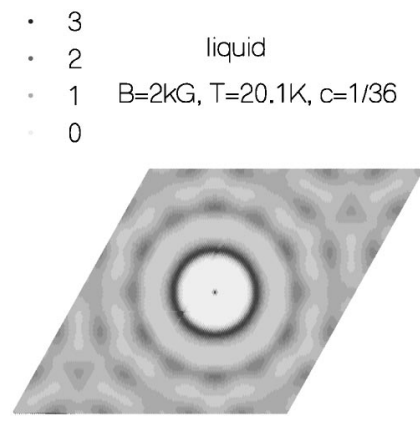

FIG. 11. Gray scale plot, as indicated, of the normalized density field $\rho(\mathbf{r}) / \rho_{0}$ at the liquid minimum for $c=1 / 36$. A pinning center is located at the center of the shown unit cell of the pin lattice.

tween the crystalline and liquid minima. This has drastic consequences for the crystallization transition. The behavior we find for $c>1 / 36(l<6)$ for both values of $B$ is significantly different from that described above. For $c=1 / 25$ and $c=1 / 16$, the apparent minima obtained in heating and cooling runs have almost the same free energy, but somewhat different values of the order parameter $m$. We have shown in Fig. 13 plots of $m$ versus $T$ obtained from heating and cooling runs for $B=2 \mathrm{kG}$ and $c=1 / 25$ (circles and squares). The small difference in the heating and cooling values of $m$ peaks at a temperature $T \equiv T_{x} \simeq 21.2 \mathrm{~K}$. In Fig. 14 we show the two corresponding density profiles obtained at $T=21.2 \mathrm{~K}$. These plots are analogous to those in Fig. 9 for $c=1 / 49$. In sharp contrast to that case, (and also to the $c=1 / 36$ case shown in Figs. 11 and 12) the two profiles are now very similar, with the one obtained in the heating run exhibiting only a slightly higher degree of order, consistent with the higher value of $m$. This leads to the suspicion that the free energy at $c=1 / 25$ may have only one very "flat" minimum near $T=T_{x}$ under the conditions studied. When attempting to find a minimum, our numerical routine stops when the free energy gradient becomes smaller than a certain small convergence parameter. When a minimum is very flat, it may stop at slightly different configurations when approaching it from different directions in configuration space.

If this situation occurs, then the density configuration at the true minimum of the free energy should be better approximated by a linear combination of the density configu-

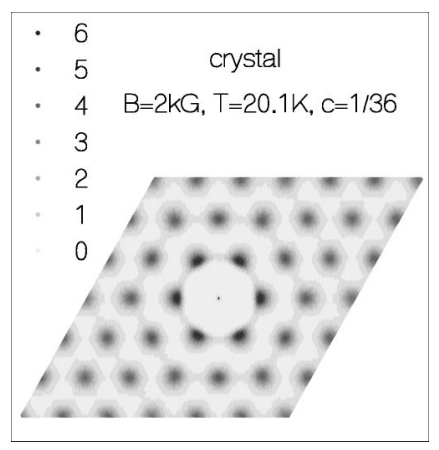

FIG. 12. Gray scale plot of the normalized density field at the crystal minimum coexisting with the liquid minimum shown in the previous figure.

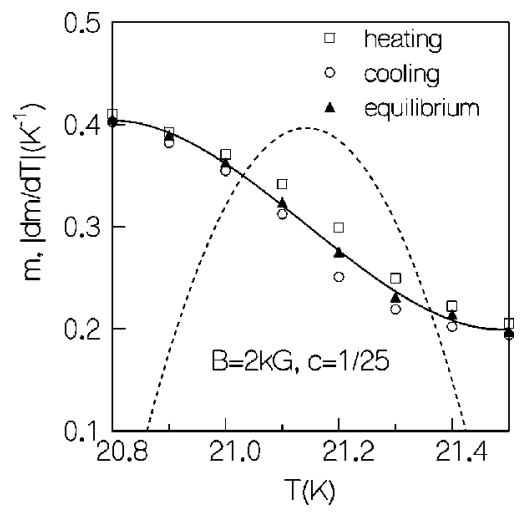

FIG. 13. Illustration of the different values of the order parameter $m$ found in heating and cooling runs (squares and circles, respectively) at higher values of the pin concentration $(c=1 / 25, B$ $=2 \mathrm{kG}$ in this case). The triangles represent the equilibrium values of $m$ found as explained in the text. The solid line is a polynomial fit to the equilibrium data. The dotted curve is the absolute value of the derivative of the solid line.

rations found in heating and cooling runs. We therefore evaluated the free energy for a set of configurations $\left\{\rho_{i}(x)\right\}$ defined by

$$
\rho_{i}(x)=x \rho_{i}^{(1)}+(1-x) \rho_{i}^{(2)},
$$

where $\left\{\rho_{i}^{(1)}\right\}$ and $\left\{\rho_{i}^{(2)}\right\}$ are the density configurations, at the same temperature, at the apparent minima obtained in heating and cooling runs, respectively. The mixing parameter $x$ is in the range $0 \leqslant x \leqslant 1$. If one then plots the free energy thus obtained either as a function of $x$ or, equivalently, as a function of $m(x) \equiv x m^{(1)}+(1-x) m^{(2)}$, where $m^{(1)}$ and $m^{(2)}$ are the order parameters in the two configurations, one finds that it indeed has a minimum at $x=x_{0} \sim 0.5$ at temperatures near the expected transition, for all higher concentrations, $c$ $\geqslant 1 / 25$. An illustration, for the case $B=2 \mathrm{kG}, T=21.2 \mathrm{~K}$, and $c=1 / 25$ discussed above, is provided by Fig. 15. If one attempts such a procedure at lower concentrations, on the other hand, the resulting plot turns out to have a maximum, rather than a minimum, near $x=0.5$.

This analysis shows that the suspicions mentioned above were correct and that for $c \geqslant 1 / 25$, only one minimum of the

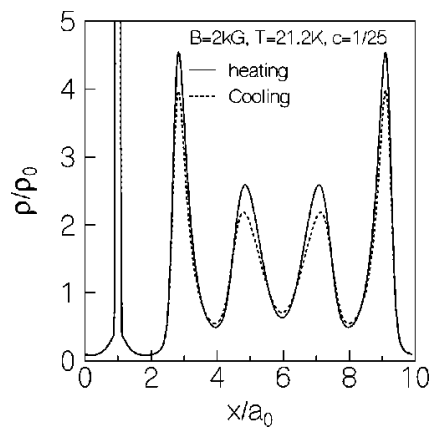

FIG. 14. Density profiles, depicted as in Fig. 9, for the heating and cooling runs shown in Fig. 13, at temperature $T=21.2 \mathrm{~K}$, which is very close to the crossover temperature $T_{x}$ defined in the text. 


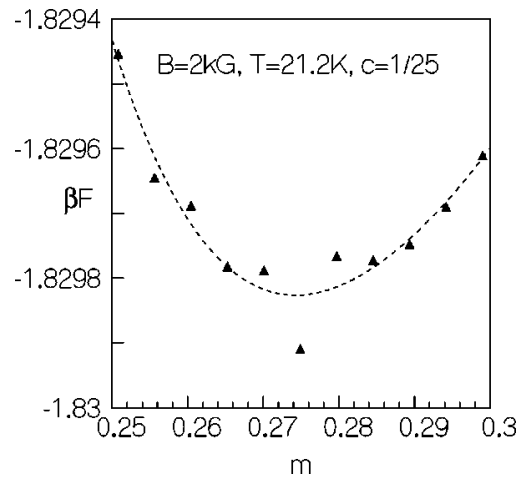

FIG. 15. The "mixed" free energy plotted as a function of $m(x)$, as explained in the text, at $c=1 / 25, B=2 \mathrm{kG}, T=21.2 \mathrm{~K}$. The triangles represent the results of the computation. The slight irregularity of the data points reflects numerical uncertainties. The dashed line is a fit to the Landau expansion of Eq. (3.3).

free energy exists at each temperature. The value of the free energy at this minimum is lower than those found in the heating and cooling runs. Thus, there is no first-order transition at $c=1 / 25$ or higher. The line of first-order transitions found for smaller values of $c$ ends at a critical point which lies between $c=1 / 36$ and $c=1 / 25$ at both values of the field considered.

At $c>1 / 36$, above the critical point, a crossover rather than a sharp transition characterizes the change from liquidlike to solidlike behavior. The crossover temperature can be conveniently defined from the numerically calculated temperature derivative of the "equilibrium" value $m\left(x_{0}\right)$ of the order parameter. Plots of both $m\left(x_{0}\right)$ and its temperature derivative are shown in Fig. 13. The temperature at which the derivative of the order parameter peaks is obviously very close to the temperature $T_{x}$ defined earlier as that at which the difference between the order parameters obtained in heating and cooling runs peaks. The crossover temperature can therefore be identified with $T_{x}$. The sharpness of the crossover suggests that $c=1 / 25, T=T_{x} \simeq 21.2 \mathrm{~K}$ is close to the critical point for $B=2 \mathrm{kG}$, as indicated by the arrow in Fig. 8 . For $c=1 / 16$ the crossover is smoother. Our results for $B$ $=3 \mathrm{kG}$ are very similar to those at the lower field, with a similar value of the critical $c$ but lower crossover temperatures (this is obvious from Fig. 8), with $T_{x} \simeq 17.6 \mathrm{~K}$ for $c$ $=1 / 25$.

The crossover to the crystal state at $c>1 / 36$ may be correlated with the onset of percolation of vortices which are "localized" according to a density-based criterion. Localization of vortices is, strictly speaking, a dynamical phenomenon, but some information about localization may be obtained from the distribution of the time-averaged local density. The local density near a point where a vortex is localized should be significantly higher than the average density $\rho_{0}$. Therefore, a density-based criterion for localization may be obtained by demanding that the density near a point where a vortex is localized exceed a suitably chosen cutoff value $\rho_{c}$. Our results for the density modulation around an isolated pinning center suggest an appropriate choice for this cutoff. One can see, for example, in Fig. 1, that in the temperature range of interest the local density in the neighbor-

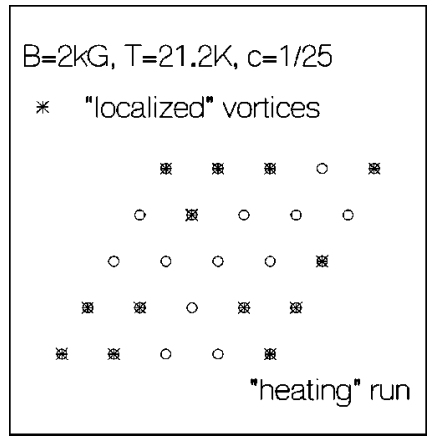

FIG. 16. Location of cells containing localized vortices under the indicated conditions. The circles denote the positions of the 25 vortex lattice unit cells contained in a unit cell of the pin lattice. A pinning center is located in the unit cell at the bottom left corner. A star in a circle denotes that the unit cell contains a "localized" vortex according to the criterion given in the text. The temperature here is slightly higher than the crossover temperature $T_{x}$ for $B$ $=2 \mathrm{kG}, c=1 / 25$. The cells containing localized vortices do not percolate across the $5 \times 5$ sample.

hood of an isolated pinning center (but outside the range of its pinning potential) does not exceed $3 \rho_{0}$ if the system is in the liquid state. This suggests that values of the local density $\rho$ less than $3 \rho_{0}$ correspond to mobile vortices. We, therefore, take $\rho_{c}=3 \rho_{0}$. We divide the computational cell into $l^{2}$ vortex-lattice unit cells and associate a localized vortex with a unit cell if the local density exceeds $\rho_{c}$ at some point inside that cell. We then examine whether the unit cells that contain localized vortices according to this criterion percolate across the sample.

All the vortex-lattice unit cells in a crystalline minimum contain localized vortices, since the maximum value of $\rho_{i}$ at the lattice sites of a crystal always exceeds $\rho_{c}$. In contrast, only the vortex-lattice unit cells in which pinning centers are located and, in some cases, the nearest neighbors of such unit cells, contain localized vortices in the coexisting liquid minimum at the crystallization transition for $c \leqslant 1 / 36$. Thus, for $c \leqslant 1 / 36$, the crystallization transition trivially coincides with a percolation of unit cells containing localized vortices. For $c \geqslant 1 / 25$, in the crossover region, we have found that the unit cells containing localized vortices do not percolate if the temperature is higher than the crossover temperature $T_{x}$ defined above, but percolation occurs below $T_{x}$. Typical results are shown in Figs. 16 and 17. In Fig. 16, we have shown the locations of the units cells containing localized vortices in the minimum obtained in the heating run for $B=2 \mathrm{kG}, T$ $=21.2 \mathrm{~K}$ and $c=1 / 25$. These unit cells do not percolate across the $5 \times 5$ computational cell, while the cells containing mobile vortices do. Since the degree of localization in the minimum obtained in the heating run is higher than that in the minimum obtained in the cooling run, no percolation would be obtained at this temperature if the cooling-run minimum or the equilibrium configuration $\rho_{i}\left(x_{0}\right)$ were used for finding the unit cells containing localized vortices. On the other hand, as shown in Fig. 17, the unit cells containing localized vortices do percolate across the sample in the "equilibrium" configuration obtained at the slightly lower $T=21.1 \mathrm{~K}$. At this temperature, the heating run shows per- 


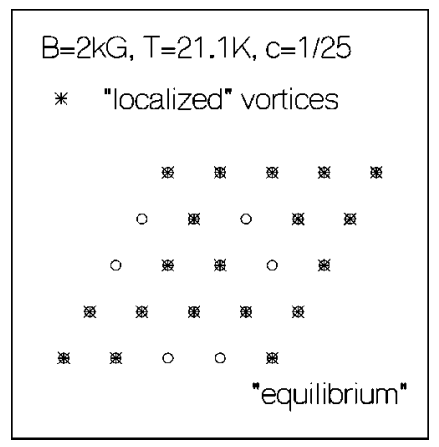

FIG. 17. As in the previous figure, but at a temperature slightly lower than the crossover temperature $T_{x}$. The cells containing localized vortices now percolate across the sample.

colation, but the cooling run does not. Thus, percolation occurs at a temperature very close to the crossover temperature $T_{x} \simeq 21.2 \mathrm{~K}$. Very similar results were obtained for $B$ $=2 \mathrm{kG}, c=1 / 16$, and $B=3 \mathrm{kG}, c=1 / 25$, indicating that this is a general condition. This result is physically reasonable: a system in which localized vortices percolate (and consequently, the mobile ones do not percolate) should behave as a "solid" at long length scales.

It is easy to see that the occurrence of a critical point in the phase diagrams of Fig. 8 does not contradict any fundamental principles. In the presence of commensurate periodic pinning, the liquid and the crystal have the same symmetry. Since the degree of order in the liquid increases with $c$, it is possible for the liquid and the crystal to become indistinguishable beyond a critical value of $c$. One can then go from one phase to the other without crossing a sharp phase boundary.

The basic features of the phase diagrams may be understood from a simple Landau theory. From well-known symmetry arguments ${ }^{42}$ one can write down a Landau expansion for $F$ :

$$
\beta F=\frac{1}{2} a_{2} m^{2}-\frac{1}{3} a_{3} m^{3}+\frac{1}{4} a_{4} m^{4}-g m,
$$

where $m$ is our order parameter, the constants $a_{3}$ and $a_{4}$ are positive, and $a_{2}$ decreases with decreasing $T$. The "ordering field" $g$ is proportional to the pin concentration $c$. A simple analysis shows that this free energy leads to a first-order transition for $g<g_{c}=a_{3}^{3} / 27 a_{4}^{2}$ and a critical point at $g=g_{c}$, $a_{2}=a_{2 c}=a_{3}^{2} / 3 a_{4}$. The transition temperature increases with the ordering field $g$ in agreement with the arguments previously discussed. The latent heat and the order parameter discontinuity $\Delta m$ vanish as $\left(g_{c}-g\right)^{1 / 2}$ as $g$ approaches $g_{c}$ from below. It was shown in Ref. 34 that our data for $\Delta s$ and $\Delta m$ are indeed well described by the form $\propto\left(c_{c}-c\right)^{1 / 2}$ with $c_{c}$ close to $1 / 25$.

More quantitatively, it is possible to fit our $\beta F$ vs $m$ data to the form Eq. (3.3). The best fit for $B=2 \mathrm{kG}, T$ $=21.2 \mathrm{~K}, c=1 / 25$, is shown in Fig. 15. The fitting parameter values are $a_{2}=70.71, a_{3}=234.87, a_{4}=261.71$, and $g$ $=7.12$. Using the values of $a_{3}$ and $a_{4}$ obtained from the fit, we get $a_{2 c}=70.26$ and $g_{c}=7.01$. These values are very close to, but slightly lower than the best-fit values of $a_{2}$ and $g$, indicating that the critical point for $B=2 \mathrm{kG}$ is, as we had already stated, very close to $c=1 / 25, T=21.2 \mathrm{~K}$. This explains the sharpness of the crossover at $c=1 / 25$. The numerical results for $B=3 \mathrm{kG}$ can be analyzed in the same way. A fit for $T=17.6 \mathrm{~K}, c=1 / 25$ yields then values of $a_{2 c}$ and $g_{c}$ which are less than $1 \%$ lower than the best-fit values of $a_{2}$ and $g$, respectively. Therefore the Landau free energy gives a good semiquantitative account of the critical behavior of our density functional computations for both values of $B$. This strengthens our conclusions about the existence and location of the critical point.

\section{SUMMARY AND DISCUSSION}

We have used in this paper numerical minimization of a discretized free energy functional to study the effects of columnar pinning on the structure and thermodynamics of a system of pancake vortices in the mixed phase of highly anisotropic layered superconductors. The most salient result of our study is the existence of a critical point in the vortexlattice melting phase diagram when a commensurate, periodic array of pinning centers is present. Our results show that the line of the melting transition in the $T-c$ plane, which is of course first order for small values of the concentration of pinning centers, terminates at a critical point as the pin concentration is increased. Beyond this critical point, the transition is replaced by a crossover, with smooth behavior of the order parameter and other thermodynamic quantities. To our knowledge, this is the first quantitative theoretical prediction of a continuous melting transition in a three-dimensional system. This critical point should be experimentally accessible: the pin lattice spacing for $B=2 \mathrm{kG}, c=1 / 25$ should be $\sim 0.55 \mu \mathrm{m}$, close to the spacing of the radiation-induced pin array of Ref. 13. The same group recently showed ${ }^{20}$ that columnar pins can be created in BSCCO in a highly controlled manner. We, therefore, expect that the fabrication of bulk HTSC samples with a periodic array of columnar pins with appropriate spacing is technically feasible. Fabrication of such samples and experiments to verify our theoretical predictions would be most welcome.

We have shown that most of the features of our phase diagram can be understood from a simple Landau theory. The critical point found in our study is analogous to the liquid-gas critical point in mean-field theory. Fluctuations are expected to change this correspondence because the symmetry of our order parameter is different from that for the liquid-gas transition. Theoretical studies of the universality class of this critical point would be interesting. However, the location of the first order melting line and the existence and experimental accessibility of the critical point should be quantitatively described by our work. We have also shown that the smooth crossover from liquid-like to solid-like behavior beyond the critical point might be interpreted as a percolation threshold for localized vortices.

The one-pin results reported here provide useful information about the dependence of the average number of vortices trapped at a pinning center on the temperature and the strength of the pinning potential, while our results for the 
interaction between two neighboring pins illustrate the occurrence of interesting effects in the liquid state arising from the commensurability of the separation of the pins with the average intervortex separation. Finally, our method yields very detailed results for the density distribution in the system, which is accessible through STM and scanning Hall probe measurements.

As noted in Sec. II, the symmetry of the threedimensional system considered here makes the calculations effectively two dimensional, with the function $\widetilde{C}$ playing the role of the two-dimensional direct pair correlation function. The direct pair correlation function of a two-dimensional vortex liquid $^{18,26}$ is quite similar to the function $\widetilde{C}$ used in the present study. We, therefore, expect that most of the results obtained here should apply, at least qualitatively, to thin-film superconductors in the presence of strong pinning centers. As noted in Sec. III, some of our one- and two-pin results are indeed in good quantitative agreement with those obtained in Ref. 18 for a two-dimensional system of vortices with strong pinning. This leads us to expect that the phase diagram obtained here for the vortex lattice melting transition in the presence of a periodic array of pins should apply, with no more than fairly minor quantitative changes, to the melting transition of a two-dimensional vortex lattice in thin-film su- perconductors with commensurate periodic pinning. Since periodic arrays of strong pinning centers have already been fabricated ${ }^{12-17}$ in thin-film superconductors, our predictions can be readily tested in experiments. One should, however, keep in mind that the predictions of our mean-field-like density functional calculation are less reliable in two dimensions where the effects of fluctuations are stronger. The melting transition in a pure two-dimensional system without pinning can be continuous, ${ }^{21}$ whereas the mean-field prediction of first-order melting is always realized in pure threedimensional systems. Our main result about the existence of a critical point in the phase diagram should apply to thin-film superconductors with commensurate periodic pinning if the system exhibits a first-order melting transition in the absence of pinning.

The melting transition of the lattice of interstitial vortices in the presence of a commensurate, periodic array of pinning centers provides a physical example of melting in the presence of an external periodic potential. Similar melting transitions are of interest in other systems such as atoms adsorbed on crystalline substrates,${ }^{21}$ and colloidal particles in interfering laser fields ${ }^{43}$ and arrays of optical traps. ${ }^{44}$ Our method and results would be of relevance to these systems also.
*Also at: Condensed Matter Theory unit, Jawaharlal Nehru Center for Advanced Scientific Research, Bangalore 560064, India.

†Electronic address: otvalls@tc.umn.edu

${ }^{1}$ For a review, see G. Blatter, M.V. Feigel'man, V.B. Geshkenbein, A.I. Larkin, and V.M. Vinokur, Rev. Mod. Phys. 66, 1125 (1994).

${ }^{2}$ L. Civale, A.D. Marwick, T.K. Worthington, M.A. Kirk, J.R. Thompson, L. Krusin-Elbaum, Y. Sun, J.R. Clem, and F. Holtzberg, Phys. Rev. Lett. 67, 648 (1991).

${ }^{3}$ R.C. Budhani, M. Sunega, and H.S. Liou, Phys. Rev. Lett. 69, 3816 (1992).

${ }^{4}$ B. Khaykovich, M. Konczykowski, K. Teitelbaum, E. Zeldov, H. Shtrikman, and M. Rappaport, Phys. Rev. B 57, R14 088 (1998).

${ }^{5}$ R.C. Budhani, W.L. Holstein, and M. Sunega, Phys. Rev. Lett. 72, 566 (1994).

${ }^{6}$ D.R. Nelson and V.M. Vinokur, Phys. Rev. B 48, 13060 (1993).

${ }^{7}$ L. Radzihovsky, Phys. Rev. Lett. 74, 4919 (1995); 74, 4923 (1995).

${ }^{8}$ A.I. Larkin and V.M. Vinokur, Phys. Rev. Lett. 75, 4666 (1995).

${ }^{9}$ R. Sugano, T. Onogi, K. Hirata, and M. Tachiki, Phys. Rev. Lett. 80, 2925 (1998).

${ }^{10}$ P. Sen, N. Trivedi, and D.M. Ceperley, Phys. Rev. Lett. 86, 4092 (2001).

${ }^{11}$ See, for example, C.J. van der Beek, M. Konczykowski, T.W. Li, P.H. Kes, and W. Benoit, Phys. Rev. B 54, R792 (1996).

${ }^{12}$ M. Baert, V.V. Metlushko, R. Jonckheere, V.V. Moshchalkov, and Y. Bruynseraede, Phys. Rev. Lett. 74, 3269 (1995).

${ }^{13}$ K. Harada, O. Kamimura, H. Kasai, T. Matsuda, A. Tonomura, and V.V. Moshchalkov, Science 274, 1167 (1996).

${ }^{14}$ J.I. Martín, M. Vélez, J. Nogués, and I.K. Schuller, Phys. Rev. Lett. 79, 1929 (1997).

${ }^{15}$ Y. Jaccard, J.I. Martín, M.-C. Cyrille, M. Vélez, J.L. Vicent, and
I.K. Schuller, Phys. Rev. B 58, 8232 (1998).

${ }^{16}$ J.I. Martín, M. Vélez, A. Hoffmann, I.K. Schuller, and J.L. Vicent, Phys. Rev. Lett. 83, 1022 (1999).

${ }^{17}$ A.N. Grigorenko, G.D. Howells, S.J. Bending, J. Bekaert, M.J. Van Bael, L. Van Look, V. V. Moshchalkov, Y. Bruynseraede, G. Borghs, I. I. Kaya, and R. A. Stradling, Phys. Rev. B 63, 052504 (2001).

${ }^{18}$ C. Dasgupta and D. Feinberg, Phys. Rev. B 57, 11730 (1998).

${ }^{19}$ C. Reichhardt, C.J. Olson, and F. Nori, Phys. Rev. B 57, 7937 (1998).

${ }^{20}$ A. Tonomura, H. Kasai, O. Kamimura, T. Matsuda, K. Harada, Y. Nakayama, J. Shimoyama, K. Kishio, T. Hanaguri, K. Kitazawa, M. Sasase, and S. Okayasu, Nature (London) 412, 620 (2001).

${ }^{21}$ D.R. Nelson and B.I. Halperin, Phys. Rev. B 19, 2457 (1979).

${ }^{22}$ M. Franz and S. Teitel, Phys. Rev. Lett. 73, 480 (1994); Phys. Rev. B 51, 6551 (1995).

${ }^{23}$ C. Reichhardt, C.J. Olson, and R.T. Scalettar, Phys. Rev. B 64, 144509 (2001).

${ }^{24}$ T.V. Ramakrishnan and M. Yussouff, Phys. Rev. B 19, 2775 (1979).

${ }^{25}$ S. Sengupta, C. Dasgupta, H.R. Krishnamurthy, G.I. Menon, and T.V. Ramakrishnan, Phys. Rev. Lett. 67, 3444 (1991).

${ }^{26}$ G.I. Menon, C. Dasgupta, H.R. Krishnamurthy, T.V. Ramakrishnan, and S. Sengupta, Phys. Rev. B 54, 16192 (1996).

${ }^{27}$ C.J. Olson, C. Reichhardt, R.T. Scalettar, G.T. Zimanyi, and N. Gronbech-Jensen, cond-mat/0008350 (unpublished).

${ }^{28}$ G. Blatter, V. Geshkenbein, A. Larkin, and H. Nordborg, Phys. Rev. B 54, 72 (1996)

${ }^{29}$ C.M. Aegerter, S.L. Lee, H. Keller, E.M. Forgan, and S.H. Lloyd, Phys. Rev. B 54, R15 661 (1996).

${ }^{30}$ S.L. Lee, C.M. Aegerter, H. Keller, M. Willemin, B. StaublePumpin, E.M. Forgan, S.H. Lloyd, G. Blatter, R. Cubitt, T.W. Li, 
and P. Kes, Phys. Rev. B 55, 5666 (1997).

${ }^{31}$ N. Nakamura, G.D. Guo, and N. Koshizuka, Phys. Rev. Lett. 71, 915 (1993).

${ }^{32}$ G.I. Menon and C. Dasgupta, Phys. Rev. Lett. 73, 1023 (1994).

${ }^{33}$ C. Dasgupta and O.T. Valls, Phys. Rev. E 62, 3648 (2000), and references therein.

${ }^{34}$ C. Dasgupta and O.T. Valls, Phys. Rev. Lett. 87, 257002 (2001).

${ }^{35}$ J. P. Hansen and I. R. McDonald, Theory of Simple Liquids (Academic, London, 1986).

${ }^{36}$ W.E. Lawrence and S. Doniach, in Proceedings of the Twelfth International Conference on Low Temperature Physics, edited by E. Kanda (Keigaku, Tokyo, 1971).

${ }^{37}$ M.V. Feigel'man, V.B. Geshkenbein, and A.I. Larkin, Physica C
167, 177 (1990).

${ }^{38}$ Note that our definition of $\Gamma$ differs by a factor of two from that in Ref. 26.

${ }^{39}$ C. Dasgupta, Europhys. Lett. 20, 131 (1992).

${ }^{40}$ C. Dasgupta and O.T. Valls, Phys. Rev. E 58, 801 (1998).

${ }^{41}$ H. Dai, S. Yoon, J. Liu, R.C. Budhani, and C.M. Lieber, Science 265, 1552 (1994).

${ }^{42}$ S. Alexander and J. McTague, Phys. Rev. Lett. 41, 702 (1978).

${ }^{43}$ Q.-H. Wei, C. Bechinger, D. Rudhardt, and P. Leiderer, Phys. Rev. Lett. 81, 2606 (1998).

${ }^{44}$ E.R. Dufresne, G.C. Spalding, M.T. Dearing, S.A. Sheets, and D.G. Grier, cond-mat/0008414 (unpublished). 